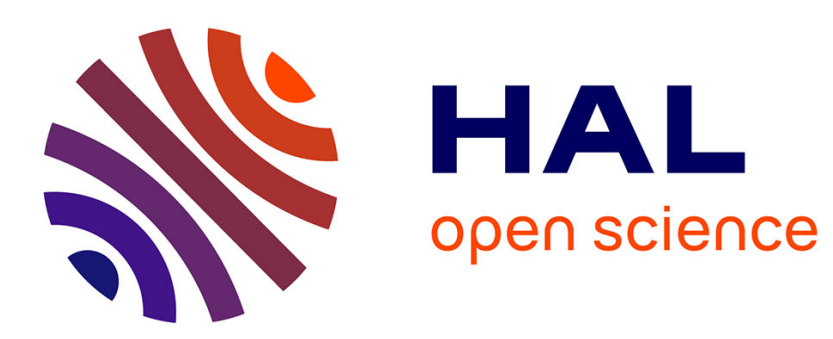

\title{
Magnetization-induced optical nonlinearity in ferromagnetic GaMnAs
}

K.-J. Han, J.-H. Kim, K.-J. Yee, X. Liu, J.K. Furdyna, François Hache

\section{To cite this version:}

K.-J. Han, J.-H. Kim, K.-J. Yee, X. Liu, J.K. Furdyna, et al.. Magnetization-induced optical nonlinearity in ferromagnetic GaMnAs. Journal of Applied Physics, 2007, 101 (6), pp.63519. 10.1063/1.2696930 . hal-00821510

HAL Id: hal-00821510

https://hal-polytechnique.archives-ouvertes.fr/hal-00821510

Submitted on 5 May 2014

HAL is a multi-disciplinary open access archive for the deposit and dissemination of scientific research documents, whether they are published or not. The documents may come from teaching and research institutions in France or abroad, or from public or private research centers.
L'archive ouverte pluridisciplinaire HAL, est destinée au dépôt et à la diffusion de documents scientifiques de niveau recherche, publiés ou non, émanant des établissements d'enseignement et de recherche français ou étrangers, des laboratoires publics ou privés. 


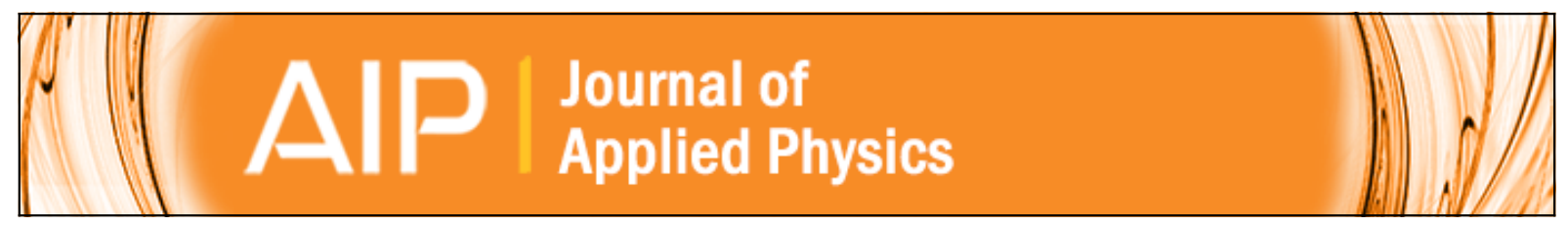

\section{Magnetization-induced optical nonlinearity in ferromagnetic GaMnAs}

Kang-Jeon Han, Ji-Hee Kim, Ki-Ju Yee, X. Liu, J. K. Furdyna, and F. Hache

Citation: Journal of Applied Physics 101, 063519 (2007); doi: 10.1063/1.2696930

View online: http://dx.doi.org/10.1063/1.2696930

View Table of Contents: http://scitation.aip.org/content/aip/journal/jap/101/6?ver=pdfcov

Published by the AIP Publishing

\section{Articles you may be interested in}

Memory effects in photoinduced femtosecond magnetization rotation in ferromagnetic GaMnAs

Appl. Phys. Lett. 94, 021101 (2009); 10.1063/1.3058765

Coherent control of magnetization precession in ferromagnetic semiconductor ( $\mathrm{Ga}, \mathrm{Mn}) \mathrm{As}$

Appl. Phys. Lett. 93, 232505 (2008); 10.1063/1.3046718

Coherent magnetization precession in GaMnAs induced by ultrafast optical excitation

Appl. Phys. Lett. 91, 112506 (2007); 10.1063/1.2785114

Carrier Dynamics and Magnetizationinduced Nonlinearity in Ferromagnetic GaMnAs

AIP Conf. Proc. 893, 1207 (2007); 10.1063/1.2730332

Magnetization manipulation in (Ga,Mn)As by subpicosecond optical excitation

Appl. Phys. Lett. 86, 152506 (2005); 10.1063/1.1899231

\section{AlP Re-register for Table of Content Alerts}

\section{Create a profile.

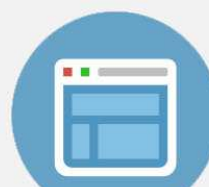 \\ Sign up today!}




\title{
Magnetization-induced optical nonlinearity in ferromagnetic GaMnAs
}

\author{
Kang-Jeon Han, Ji-Hee Kim, and Ki-Ju Yee ${ }^{\text {a) }}$ \\ Department of Physics, Chungnam National University, Daejeon 305-764, Korea \\ X. Liu and J. K. Furdyna \\ Department of Physics, University of Notre Dame, Notre Dame, Indiana 46556 \\ F. Hache \\ Laboratoire d'Optique et Biosciences, CNRS/Ecole Polytechnique, 91128 Palaiseau Cedex, France
}

(Received 6 November 2006; accepted 4 January 2007; published online 23 March 2007)

\begin{abstract}
We report the observation of a coherent nonlinear signal in pump-probe experiments on a ferromagnetic GaMnAs. The coherent signal, which is originating due to coherent interaction between pump and probe beams, depends on the polarization configuration of each beam and follows the sample magnetization as it changes with the applied magnetic field and/or the sample temperature. (C) 2007 American Institute of Physics. [DOI: 10.1063/1.2696930]
\end{abstract}

\section{INTRODUCTION}

Ferromagnetic semiconductor (FMS) $\mathrm{Ga}_{1-x} \mathrm{Mn}_{x} \mathrm{As}$ continues to attract much interest due to its potential applications in spintronics, in which the spin of the material is expected to provide an additional degree of freedom in electronic applications. ${ }^{1,2}$ With increasing Mn concentration $x$ in asgrown $\mathrm{Ga}_{1-x} \mathrm{Mn}_{x} \mathrm{As}$ samples, both the Curie temperature $T_{C}$ and the hole concentration $p$ have been shown to increase up to around $x=0.06$, beyond which point $T_{C}$ and $p$ level off and decrease, primarily due to the formation of Mn interstitials and $\mathrm{Mn}$ precipitates. ${ }^{3}$ The Curie temperature can be enhanced, however, by increasing the hole density and/or the total magnetic moment of the material by postgrowth annealing or by modulation doping. ${ }^{4,5}$ The magnetic properties in FMSs can be probed by several experimental methods, including magnetotransport, magnetic circular dichroism (MCD), and superconducting quantum interference device (SQUID) magnetometry. ${ }^{6,7}$

Recently it has been shown that sample magnetization (spontaneous or induced by an applied magnetic field) can produce magnetism-induced nonlinear optical signals. This is largely because magnetization in the material breaks the time-reversal symmetry - the same mechanism as that responsible for magneto-optical Kerr effect, magnetic circular dichroism, and Faraday rotation. Magnetization-induced second harmonic generation (SHG) was reported for ferromagnetic material surfaces and for a polar ferromagnet, space inversion symmetry being broken in both cases. ${ }^{8,9}$ Magneticfield induced SHG was also observed for the semiconductor GaAs and for a two-sublattice antiferromagnetic compound $\mathrm{CuB}_{2} \mathrm{O}_{4} \cdot{ }^{10,11}$ In the case of FMS materials, extremely large magnetization-induced SHG was reported for GaMnAs. ${ }^{12}$ In this paper, we report the observation of a magnetizationinduced coherent nonlinear signal in pump-probe experiments for ferromagnetic GaMnAs.

In a pump-probe experiment, an excitation beam $E_{e}(t)$ is sent on the sample together with a delayed probe beam

${ }^{a)}$ Electronic mail: kyee@cnu.ac.kr
$E_{p}(t-\tau)$, where $\tau$ is the variable delay time between the two beam pulses. Here both $E_{e}(t)$ and $E_{p}(t-\tau)$ are the slowly varying envelopes of electric fields for an implicit $e^{-i \omega t}$ time dependence, where $\hbar \omega$ corresponds to the central photon energy of pump and probe beams. Introducing a response function $A(t)$, the change in the transmitted probe energy is composed of the following two terms: ${ }^{13-15}$

$$
\Delta E_{p}(\tau) \propto \gamma(\tau)+\beta(\tau),
$$

where

$$
\begin{aligned}
\gamma(\tau)= & \int_{-\infty}^{+\infty} d t \int_{-\infty}^{+\infty} d t^{\prime} E_{p i}^{*}(t-\tau) E_{p j}(t-\tau) E_{e k}^{*}\left(t^{\prime}\right) \\
& \times E_{e l}\left(t^{\prime}\right) A_{i j k l}\left(t-t^{\prime}\right)
\end{aligned}
$$

is a noncoherent contribution, and

$$
\begin{aligned}
\beta(\tau)= & \int_{-\infty}^{+\infty} d t \int_{-\infty}^{+\infty} d t^{\prime} E_{p i}^{*}(t-\tau) E_{e j}(t) E_{e k}^{*}\left(t^{\prime}\right) \\
& \times E_{e l}\left(t^{\prime}-\tau\right) A_{i j k l}\left(t-t^{\prime}\right)
\end{aligned}
$$

is a coherent term. The coherent term $\beta(\tau)$, often called a coherent artifact (CA), is induced by coherent interaction between the pump and the probe beams during the overlap of the two beam pulses, while the incoherent term $\gamma(\tau)$ represents the convolution of the material response with the pulse intensity autocorrelation. ${ }^{16} \mathrm{We}$ demonstrate that the observed coherent signal in GaMnAs which is related to $\beta(\tau)$ principally originates from magnetization-induced coherent interactions between pump and probe beams, based on the dependence of the signal on magnetic field, sample temperature, as well as on beam polarizations and on the photon wavelength.

\section{EXPERIMENT}

We have performed degenerate pump-probe measurements on a ferromagnetic GaMnAs layer using a Kerr-lens mode-locked Ti:sapphire laser. The laser generates transformed-limited pulses with full width at half maximum (FWHM) pulse durations of $50 \mathrm{fs}$ at a repetition rate of $90 \mathrm{MHz}$. Center wavelength of the laser was tunable from 
760 to $840 \mathrm{~nm}$ by controlling the slit between the intracavity prism pair. The pulses are divided into intense pump pulses and weak probe pulses by a beam splitter. The pump fluence was measured to be around $0.1 \mathrm{~mJ} / \mathrm{cm}^{2}$, considering the focused spot diameter of about $30 \mu \mathrm{m}$. In standard pumpprobe measurements the pump beam excites a sample, creating a nonequilibrium distribution of electrons and holes, and the probe beam is used to measure the process of carrier relaxation back to equilibrium by detecting transmission or reflection changes in the probe beam as a function of time delay between the pump and the probe pulses.

\section{RESULTS AND DISCUSSION}

We have measured time-resolved reflection changes on a ferromagnetic GaMnAs layer in the presence of magnetic fields. Magnetic fields up to a $0.15 \mathrm{~T}$ were generated by an electromagnet, with the field direction parallel to the sample growth direction (Faraday geometry). The angle between pump beam and probe beam was about $5^{\circ}$ with both beams incident almost normal to the sample surface. Polarization of the pump and the probe beams was independently adjusted to right-handed $(\mathrm{C}+)$ or left-handed $(\mathrm{C}-)$ circular polarization by $\lambda / 4$ wave plates placed in the paths of the pump and the probe beams. The sample was put inside a continuous-flow cryostat and temperature dependence of the signal was measured down to $10 \mathrm{~K}$.

A $\mathrm{Ga}_{1-x} \mathrm{Mn}_{x}$ As layer with Mn concentration $x$ of 0.059 was studied in this work. The layer was grown by molecular beam epitaxy (MBE) on a semi-insulating (001) GaAs substrate in a Riber R\&D MBE machine. The Mn concentrations $x$ were deduced from the Mn cell temperature that has been previously calibrated by $\mathrm{x}$-ray diffraction studies of $\mathrm{Ga}_{1-x} \mathrm{Mn}_{x} \mathrm{As} / \mathrm{GaAs}$ epilayers. The hole concentration of the sample was estimated to be $4.8 \times 10^{19} \mathrm{~cm}^{-3}$ by measuring the Hall resistance up to $6 \mathrm{~T}$ at room temperature, where the contribution from the anomalous Hall effect, while not negligible, is considerably reduced. The contribution from anomalous Hall effect may have generated larger hole density than the actual value, as the ordinary Hall resistance and the anomalous Hall resistance will have the same trend under magnetic fields. Magnetotransport measurements on the sample indicate typical ferromagnetic behavior, with a Curie temperature of $54 \mathrm{~K}$.

Figure 1 shows transient probe beam intensity changes induced by the pump beam measured in reflection geometry at a center wavelength of $810 \mathrm{~nm}$. A magnetic field of $1.3 \mathrm{kOe}$ was applied along the sample growth direction, and the measurements were performed at a sample temperature of $10 \mathrm{~K}$ with different circular pump and probe beam polarizations. The reflective pump-probe signals can be decomposed into the coherent part near time delay zero and the incoherent part that is slowly varying compared to the coherent component. The inset shows time-resolved Kerr ellipticities $\left(\Delta \varepsilon_{K}\right)$ that are obtained by subtracting the transient reflection signal for probe polarization at $\mathrm{C}+$ from that at $\mathrm{C}-$ polarization. The overal similar behavior of the Kerr ellipticities at different pump polarizations indicates that the slowly varying Kerr signal is mainly coming from the laser-

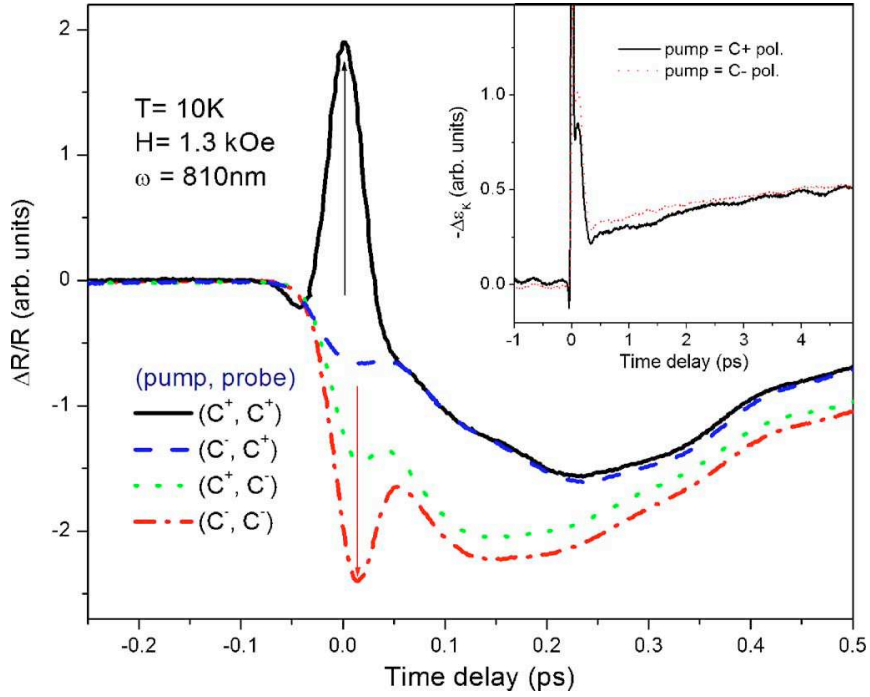

FIG. 1. (Color online) Transient probe beam reflection change as a function of time delay between pump and probe beams for different combinations of pump/probe circular polarizations, indicated by $\mathrm{C}+(\mathrm{C}-)$ for right-handed (left-handed) circular polarizations. The sample temperature is $10 \mathrm{~K}$ and the center wavelength is $810 \mathrm{~nm}$. The data were taken in a magnetic field of $1.3 \mathrm{kOe}$ applied normal to the plane of the GaMnAs layer. The inset curves show Kerr ellipticities $\left(\Delta \varepsilon_{K}\right)$ at different pump polarizations.

induced magnetization quenching effect. ${ }^{17}$ We note that the fast dynamics in $\Delta \varepsilon_{K}$ observed before $0.5 \mathrm{ps}$ is similar to the observation by Wang et al., where they interpreted it as spin heating effect through $s p-d$ exchange interaction between photocarriers and Mn ions. ${ }^{18,19}$ What is interesting to us is the dependence of the coherent component on the polarizations of both the pump and the probe beams. Under the applied magnetic field, a positive coherent peak signal is observed near zero time delay at the (pump, probe) polarization of $(\mathrm{C}+, \mathrm{C}+)$, where $\mathrm{C}+(\mathrm{C}-)$ correspond to right-handed (left-handed) circular polarization. On the contrary, a negative coherent peak is observed in the $(\mathrm{C}-, \mathrm{C}-)$ configuration; and the coherent signal is much smaller in polarization configurations $(\mathrm{C}+, \mathrm{C}-)$ and $(\mathrm{C}-, \mathrm{C}+)$. The dependence of the coherent signal on both the pump and the probe polarizations indicates that it is originating from the mutual interaction between the pump and the probe photons. However, from Ref. 15, one obtains that in a nongyrotropic medium, the signals for $(\mathrm{C}+, \mathrm{C}+)$ and $(\mathrm{C}-, \mathrm{C}-)$ conditions are expected to be equal, the same being true for $(\mathrm{C}+, \mathrm{C}-)$ and $(\mathrm{C}-, \mathrm{C}+)$ signals. This is clearly not the case in Fig. 1. This feature is a clear indication that most of the signal originates from a gyrotropic effect in the sample. In our GaMnAs samples, this gyrotropy is likely to be induced by the applied magnetic field, as already observed in MCD. ${ }^{7}$

In order to investigate the origin of the dependence of the coherent interaction signal on circular polarization in pump-probe measurements, we have changed the magnetic field strength and direction in the $(\mathrm{C}+, \mathrm{C}+)$ configuration. The sample temperature and the photon wavelength were kept at $10 \mathrm{~K}$ and $810 \mathrm{~nm}$, respectively. Figure 2(a) shows transient reflection changes of the probe beam at various magnetic fields along the growth direction, which constitute the magnetic hard axis of the studied layer. As shown in the figure, the positive coherent artifact signal at positive mag- 

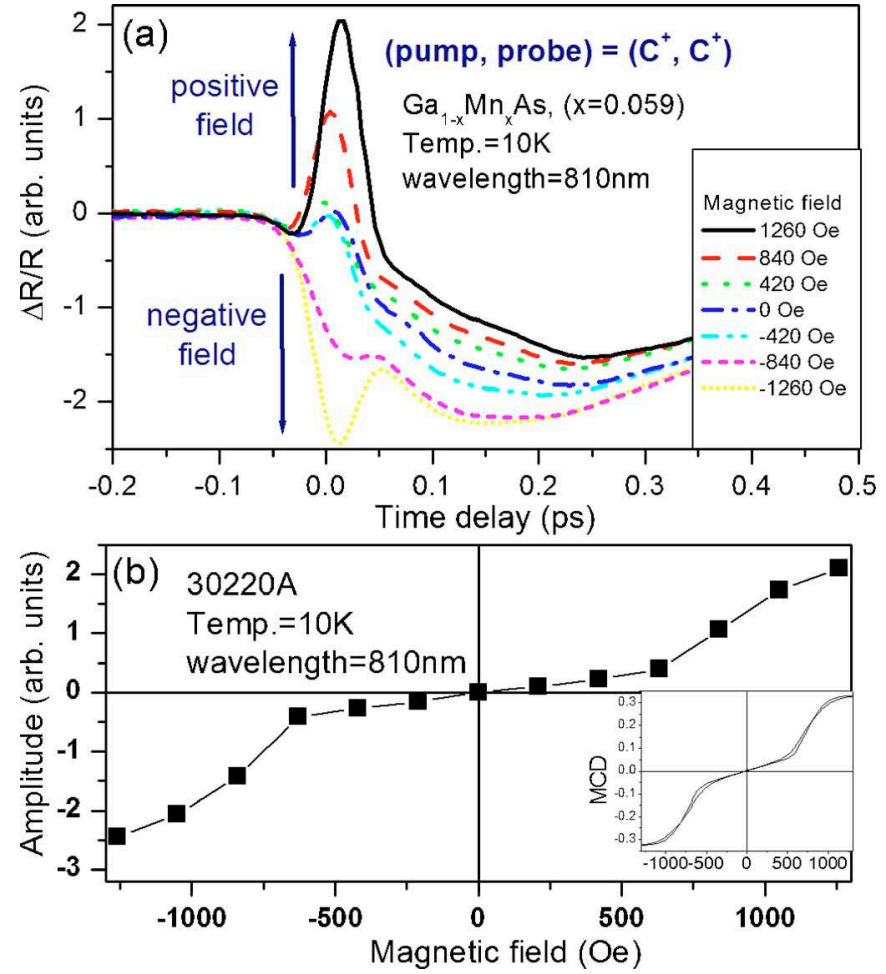

FIG. 2. (Color online) (a) Transient reflection changes for several magnetic fields observed at the following experimental conditions: temperature is $10 \mathrm{~K}$, center wavelength is $810 \mathrm{~nm}$, and polarization configuration is $(\mathrm{C}+, \mathrm{C}+)$. (b) Amplitude of coherent signal near zero time delay as a function of magnetic field. The vertical scale was shifted such that the amplitude of the coherent artifact at zero field corresponds to zero.

netic fields decreases as the field is decreased, and the sign of the coherent signal reverses when the applied field is increased in the opposite direction. In Fig. 2(b) we have plotted the amplitude of the coherent interaction signal as a function of the applied magnetic field, where the amplitude was obtained by subtracting the background signal from the incoherent contributions. The zero of the vertical axis was shifted so as to set the peak of the coherent artifact to zero at zero magnetic field. As we compare the magnetic field dependence of the coherent signal amplitude with MCD, which is a measure of the sample magnetization in FMSs [see inset of Fig. 2(b)], the amplitude of the coherent artifact signal is clearly seen to follow the sample magnetization. We note that the two-slope behaviors under magnetic fields both in $\mathrm{MCD}$ and in coherent signal are most likely due to the magnetic anisotropy parameters of the GaMnAs layer.

We have also measured the temperature dependence of the coherent artifact signal at a fixed polarization configuration $(\mathrm{C}+, \mathrm{C}+)$. Figure 3(a) shows transient pump-probe signals measured at several temperatures at magnetic fields +1260 and -1260 Oe. As shown in Fig. 3(b), the coherent signal amplitude which is obtained by subtracting the negative field signal from the positive one decreases with increased sample temperature and becomes negligible above the Curie temperature. This type of temperature dependence is what would be expected for the magnetization of the sample. Thus, these data again indicate that the coherent signal is induced by magnetization-induced coherent interactions in ferromagnetic GaMnAs.
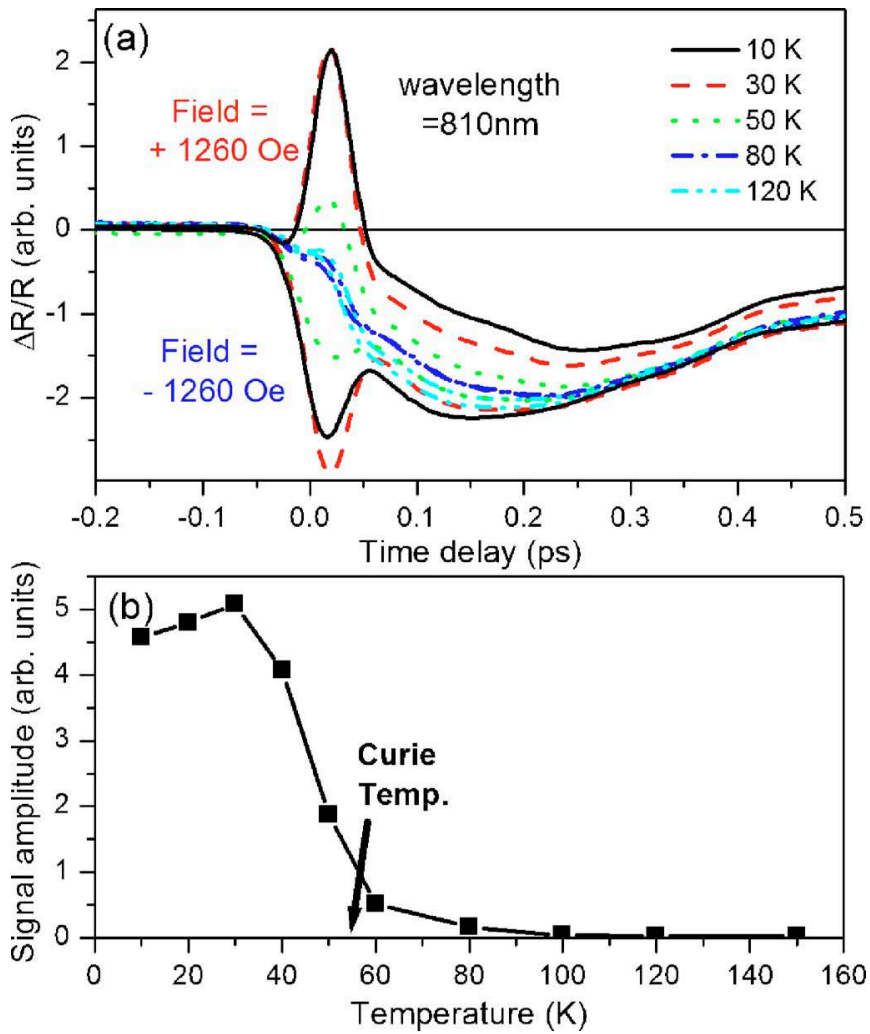

FIG. 3. (Color online) (a) Transient reflection changes at different sample temperatures observed at +1260 and $-1260 \mathrm{G}$, at center wavelength $810 \mathrm{~nm}$, and polarization configuration $(\mathrm{C}+, \mathrm{C}+)$. (b) Amplitude of coherent artifact signal as a function of applied sample temperature. The amplitude value was obtained by subtracting the signal at $-1260 \mathrm{G}$ from that at $+1260 \mathrm{G}$.

As we change the center wavelength of the pulses used for the pump-probe experiments, the amplitude of the coherent artifact signal is observed to change its sign even if the polarization and magnetic field remain unchanged. Figure 4 shows the coherent artifact amplitude as a function of the center wavelength measured at a polarization configuration $(\mathrm{C}+, \mathrm{C}+)$ at $10 \mathrm{~K}$. The signal is negative for wavelengths from 760 to $790 \mathrm{~nm}$ and becomes positive for wavelengths

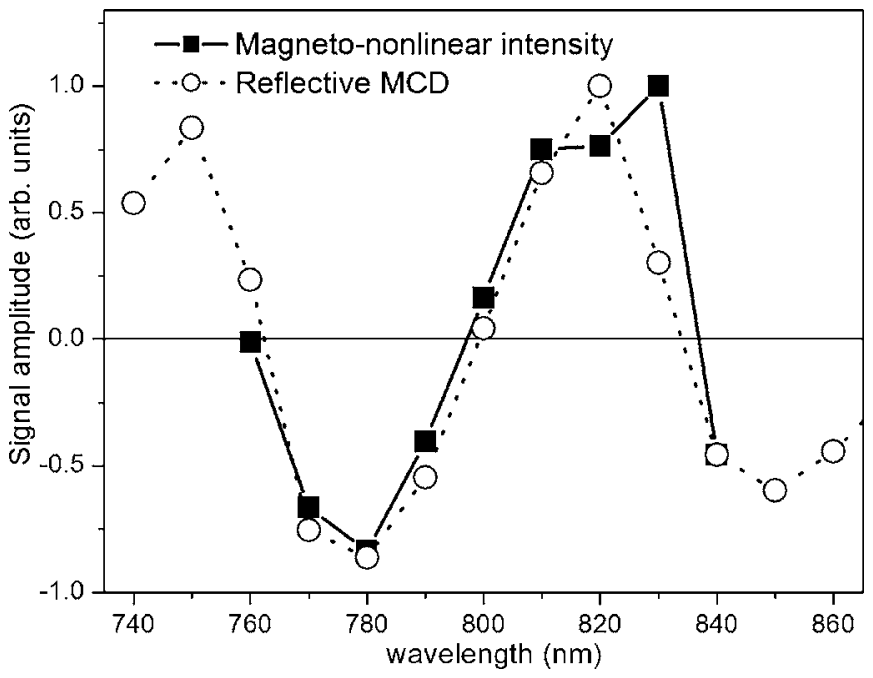

FIG. 4. The amplitude of the coherent signal (full squares) and MCD (open circles) as a function of pulse center wavelength. 
from 810 to $830 \mathrm{~nm}$. The wavelength dependence of the sign of the coherent artifact signal is similar to that of the MCD spectra, also plotted in Fig. 4. The observed dependences of the coherent artifact signal on the magnetic field and the temperature strongly suggest that the coherent signal observed in pump-probe measurements near zero time delay originates from the magnetization-induced coherent interactions between the pump and the probe beams. This conclusion is also borne out by the MCD data, which are intimately related to the sample magnetization. The similarity between the coherent signal and MCD is a further indication that both effects are intimately related. This relation is to be sought in the microscopic origin of the magnetic-field-induced phenomena. $^{20}$ In MCD, the effect relies on interaction of light with two electric dipole moments and interaction of the static magnetic field with one magnetic dipole moment. Similarly, the gyrotropic effect in the pump-probe experiment is the result of interaction of light with four electric dipole moments and interaction of the static magnetic field with one magnetic dipole moment. Our measurements therefore confirm that the coherent pump-probe response in GaMnAs is dominated by magnetic-field-induced gyrotropic effects.

\section{CONCLUSIONS}

We have performed degenerate pump-probe measurements on ferromagnetic GaMnAs in an applied magnetic field. The coherent signal which exists when the pump and the probe beams overlap temporally is seen to closely follow the magnetization of the GaMnAs layer as we changed the magnetic field and the sample temperature. This feature allows us to ascertain the origin of this coherent signal as mainly due to a magnetization-induced gyrotropy very similar to what is observed in MCD. We note that this behavior of the coherent signal can be used as another method for probing the magnetic properties of ferromagnetic semiconductors.

\section{ACKNOWLEDGMENTS}

This work was supported by the KOSEF (Grant No. R01-2005-000-10616-0), the Korea Research Foundation Grant (KRF-2004-041-C00117), and the U.S. National Science Foundation Grant Nos. DMR02-10519 and DMR0603762 .

${ }^{1}$ H. Ohno, M. Munekata, T. Penney, S. von Molnar, and L. Chang, Phys. Rev. Lett. 68, 2664 (1992).

${ }^{2}$ H. Ohno, A. Shen, F. Matsukura, A. Oiwa, A. Endo, S. Katsumoto, and Y. Iye, Appl. Phys. Lett. 69, 363 (1996).

${ }^{3}$ H. Ohno, J. Magn. Magn. Mater. 200, 110 (1999).

${ }^{4}$ K. C. Ku et al., Appl. Phys. Lett. 82, 2302 (2003).

${ }^{5}$ T. Wojtowicz et al., Appl. Phys. Lett. 83, 4220 (2003).

${ }^{6}$ H. Ohno, H. Munekata, S. von Molnar, and L. L. Chang, J. Appl. Phys. 69, 6103 (1991)

${ }^{7}$ K. Ando, T. Hayashi, M. Tanaka, and A. Twardowski, J. Appl. Phys. 83, 6548 (1998)

${ }^{8}$ M. Fiebig, Th. Lottermoser, D. Fröhlich, A. V. Goltsev, and R. V. Pisarev, Nature (London) 419, 818 (2002).

${ }^{9}$ Y. Ogawa, Y. Kaneko, J. P. He, X. Z. Yu, T. Arima, and Y. Tokura, Phys. Rev. Lett. 92, 047401 (2004).

${ }^{10}$ V. V. Pavlov, A. M. Kalashnikova, R. V. Pisarev, I. Sänger, D. R. Yakovlev, and M. Bayer, Phys. Rev. Lett. 94, 157404 (2005).

${ }^{11}$ R. V. Pisarev, I. Sänger, G. A. Petrakonskii, and M. Fiebig, Phys. Rev. Lett. 93, 037204 (2004).

${ }^{12}$ Y. Ogawa, H. Akinaga, F. Takano, T. Arima, and Y. Tokura, J. Phys. Soc. Jpn. 73, 2389 (2004).

${ }^{13}$ B. S. Wherrett, A. L. Smirl, and T. F. Boggess, IEEE J. Quantum Electron. QE19, 680 (1983)

${ }^{14}$ T. F. Heinz, S. L. Palfrey, and K. B. Eisenthal, Opt. Lett. 9, 359 (1984).

${ }^{15}$ M. Mesnil, M. C. Schanne-Klein, and F. Hache, Eur. Phys. J. D 5, 447 (1999).

${ }^{16}$ E. P. Ippen and C. V. Shank, in Ultrafast Light Pulses, Topics in Applied Physics Vol. 18, edited by S. L. Shapiro, (Springer-Verlag, Berlin, 1977).

${ }^{17}$ A. V. Kimel, G. V. Astakhov, G. M. Schott, A. Kirilyuk, D. R. Yakovlev, G. Karczewski, W. Ossau, and G. Schmidt, Phys. Rev. Lett. 92, 237203 (2004).

${ }^{18}$ J. Wang, C. Sun, J. Kono, A. Oiwa, H. Munekata, L. Cywinski, and L. J. Sham, Phys. Rev. Lett. 95, 167401 (2005).

${ }^{19}$ J. Wang et al., J. Phys.: Condens. Matter 18, R501 (2006).

${ }^{20} \mathrm{G}$. H. Wagnière, Linear and Nonlinear Optical Properties of Molecules (VCH, Weinheim, 1993), p. 56. 